\title{
ACTOS DE HABLA DIRECTIVOS EN EL ALTA HOSPITALARIA
}

\author{
Susana GALLARDO \\ Universidad de Buenos Aires (Argentina)
}

\section{RESUMEN}

El propósito de este trabajo es aportar al conocimiento de la interacción médico-paciente durante el alta hospitalaria a través del análisis de los actos de habla directivos formulados por los profesionales. Se intenta determinar los distintos tipos de actos de habla directivos que se formulan y la forma en que se realizan. El marco teórico es el de la lingüística textual (Ciapuscio et al. 2010), con el aporte del enfoque cognitivo propuesto por Pérez Hernández (2001), incluyendo el análisis léxico-gramatical de los verbos que designan la acción o el comportamiento propuesto por el directivo. El corpus se compone de seis interacciones orales entre médicos y pacientes que sufrieron infarto y fueron sometidos a cirugía. Se identificaron cuatro tipos de directivos según el tipo de predicado y el campo de aplicación de las acciones propuestas. Los directivos difieren en el grado de mitigación y de opción ofrecida al destinatario. Se puede afirmar que la forma en que se realizan los directivos en el momento del alta se vincula al costo-beneficio de las acciones propuestas, y a la voluntad del especialista en que el paciente lleve a cabo las acciones y actividades propuestas. Además de los procedimientos de mitigación, los profesionales despliegan estrategias corteses mostrando empatía y reforzando la solidaridad y la imagen social de autonomía y afiliación de los pacientes.

PALABRAS CLAVE: interacción médico-paciente, actos de habla directivos, mitigación, estrategias corteses.

\section{AbSTRACT}

This paper aims to contribute to the knowledge of doctor-patient interaction during hospital discharge through the analysis of directive speech acts uttered by doctors. At a higher level of granularity, the focus of this paper is on the different types of direct speech acts performed and the way(s) how these are performed. We adopt the perspective of text linguistics (Ciapuscio et al. 2010), including the cognitive approach proposed by Pérez Hernández (2001). We also include a lexico-grammatical analysis of those verbs which refer to actions or behavior proposed by the directive speech act. The corpus used in this study consists of six 
oral interactions between doctors and patients who suffered heart attack and underwent surgery. We show that four types of directives can be identified according to the scope of application and the type of verbs. Directive speech acts are shown to differ in their degree of optionality as well as their degree of mitigation. It can be stated that the way in which directives are realized by doctors in hospital discharge is associated with the cost-benefit of the activities in question as well as the doctor's willingness to achieve patient adherence to advice. Moreover, doctors display politeness strategies to reinforce solidarity and the social image of patients.

KEYWORDS: doctor-patient interaction, directive speech acts, mitigation, politeness strategies.

Fecha de recepción: 16/01/2019

Fecha de aceptación: 15/03/2019

Fecha de la versión definitiva: 27/05/2019

\section{INTRODUCCIÓN}

En las últimas décadas, numerosas investigaciones desde diversas perspectivas, como el análisis conversacional, la etnografía de la comunicación y la sociolingüística, se han centrado en la interacción entre médicos y pacientes en la consulta médica (Cicourel 1985, 2005; Fisher 1984; Fisher y Groce 1985; Drass 1982; Aronsson y Sätterlund-Larsson 1987; AinsworthVaughn 2003; Fleischman 2003; Gülich 2003, entre otros). Algunos de estos trabajos han puesto en evidencia las diferencias de estatus y poder que entran en juego en esas interacciones; y la asimetría en cuanto a la prioridad del médico para formular preguntas e iniciar y finalizar el encuentro (Ainsworth-Vaughn 2003). Sin embargo, los cambios sociales producidos en los últimos tiempos han modificado el modelo tradicional asimétrico de la relación médico-paciente, y han preparado el terreno para un debilitamiento de las jerarquías a favor de los pacientes (Černý 2010a). Este autor observó también que la relación de simetría-asimetría puede variar a lo largo de la consulta.

Por otra parte, en estudios en lengua española desde la perspectiva de la sociopragmática, se han indagado las estrategias de atenuación y cortesía en el consejo médico y se ha observado que la función de estas estrategias no siempre es la de mitigar los riesgos para la imagen del paciente (Cepeda 2005; Rodríguez Tembrás 2016; Bañón Hernández 2017; Hernández Flores y Rodríguez Tembrás 2018, entre otros). De hecho, el que se produzca o no el riesgo para la imagen depende de factores como el contexto de la interacción, la naturaleza del consejo y la autoridad del emisor, entre otros. Hay casos en que el consejo puede reforzar la imagen de afiliación del paciente, al sentirse bien atendido por el médico. 
La formulación de actos directivos es un aspecto característico de la interacción médico-paciente, y ha recibido la atención de diversos investigadores. La forma de expresar los directivos, y la cantidad de información brindada, contribuyen a la aceptación y adherencia del paciente al tratamiento (West 1990; Heritage y Sefi 1992). Por su parte, Černý (2007) observó que los directivos tienden a formularse en forma directa durante la etapa de examen del paciente, e indirecta, en la etapa del tratamiento.

Los actos de habla directivos, que expresan la intención del hablante de lograr que el destinatario lleve a cabo una acción, comprenden órdenes, pedidos y súplicas, así como recomendaciones, consejos e instrucciones. Dentro de esta variedad, se han propuesto criterios para marcar distinciones, por ejemplo, en función del beneficiario de la acción propuesta, se puede distinguir entre directivos impositivos y no impositivos (Haverkate 1984); el primer grupo incluye actos cuyo beneficiario es el hablante (órdenes, ruegos y pedidos). Los no impositivos -consejos, sugerencias e instruccionestienen como beneficiario al destinatario. No obstante, las diferentes ilocuciones directivas no constituyen clases clara y objetivamente delimitables sino que existen solapamientos entre las distintas categorías (Gras y García 2010; Montolío y López Samaniego 2010).

En general, los estudios acerca de la interacción médico-paciente se han centrado en la consulta médica, es decir, la instancia en la cual un paciente solicita la atención de un profesional de la salud para obtener diagnóstico y tratamiento. Se ha postulado una estructura para este género discursivo, que consiste en apertura, relato de los síntomas, examen del paciente, diagnóstico, prescripción y consejos, y cierre (Ten Have 1989; Bagheri et al. 2015, entre otros). En la consulta, se ha señalado que el médico realiza tres tipos de actos de habla: interrogar, dar indicaciones e informar (Coulthard y Ashby 1975; Černý 2010a, 2010b, 2014).

Existe otra instancia de interacción médico-paciente a la que no se le ha prestado tanta atención: es la que acontece en el momento del alta hospitalaria, luego de la internación. Los trabajos acerca del alta hospitalaria, provenientes del ámbito médico, destacan que esta interacción es un proceso complejo, orientado a prevenir la readmisión innecesaria, la cual genera altos costos para los sistemas de salud y afecta la calidad de vida de los pacientes; además, se ha puesto énfasis en la necesidad de informar al paciente por qué ha estado internado, cómo podrá manejarse en su casa y qué hacer en caso de problemas (Alper et al. 2017; Rush et al. 2016). A pesar de su importancia, la interacción durante el alta hospitalaria ha recibido poca atención desde una perspectiva lingüística y discursiva.

El propósito de este trabajo es aportar al conocimiento de la interacción médico-paciente durante el alta hospitalaria, proponiendo, por un lado, una estructura para este género discursivo. Por el otro, a través del análisis 
de los actos de habla directivos que formulan los médicos en esta instancia, nos proponemos realizar la caracterización de los distintos tipos en función de determinados parámetros como el grado de mitigación, el grado de opción que brindan, y el costo-beneficio de las acciones propuestas.

\section{MARCO TEÓRICO}

Este trabajo se realiza desde la perspectiva general de la lingüística textual, considerando los actos directivos en su realización en un texto concreto, y tomando como variables las informaciones específicas de las diferentes dimensiones: funcional, situacional, temática y léxico-gramatical (Ciapuscio et al. 2010). Asimismo, en el análisis de las ocurrencias de actos directivos, se sigue el enfoque cognitivo propuesto por Pérez Hernández (2001), quien, para cada directivo, propone un modelo cognitivo que incluye información sobre los participantes en la interacción, sus relaciones y el contexto en que se lleva a cabo. Esa información se traduce en un conjunto de variables graduales, que conforman el Modelo Cognitivo Idealizado (MCI), sobre la base del postulado por Lakoff (1987). Los MCI son entidades conceptuales, estructuradas, con las que organizamos nuestro conocimiento. El hecho de que las variables sean graduales permite diferentes grados de implementación y, por lo tanto, habilita al analista para dar cuenta de miembros más o menos prototípicos de una categoría ilocutiva particular (Searle 1969).

Las variables propuestas por Pérez Hernández (2001: 80) son las siguientes:

1. Tipo de agente: la persona que lleva a cabo la acción expresada en la predicación, puede ser el hablante, el destinatario o un tercero. En este caso, el agente es el paciente, y la 'fuente modal' -quien propone su realización- (Verstraete 2005) es el médico.

2. Tiempo de la acción propuesta: pasado, presente o futuro. Según Searle (1969), una condición de los actos directivos es que el contenido proposicional refiera a una acción futura.

3. Grado de capacidad del agente para realizar la acción expresada en la predicación. Esta capacidad debe ser presupuesta por el hablante; de hecho, es una de las condiciones preparatorias para la realización de un pedido o una orden (Searle 1969).

4. Grado de voluntad del hablante: su deseo de que tenga lugar el estado de cosas expresado en la predicación. Pérez Hernández (2001) señala que, si el beneficiario es el hablante, es lógico que sea alto su grado de voluntad para que la acción se realice. Si bien en nues- 
tro caso el beneficiario es el destinatario, podemos inferir que es alta la voluntad del médico para que su paciente realice las acciones propuestas y así pueda recuperar su salud. Si bien esta variable se basa en supuestos sobre los comportamientos humanos, los hablantes pueden dar indicios de su grado de voluntad en que se cumplan las acciones, por ejemplo, dando una orden o consejo como si fuera un pedido y simulando que la realización de la acción es para su beneficio.

5. Grado de voluntad del destinatario: su deseo de que tenga lugar el estado de cosas expresado en la predicación. Tanto la variable de voluntad del hablante como del destinatario se basan en suposiciones en relación con el costo beneficio de la acción propuesta. Según Pérez Hernández ( op. cit.), en una orden prototípica, cuyo beneficiario es el hablante, puede que la voluntad del destinatario sea baja. En cambio, en un consejo, si el beneficio es para el destinatario, se supone que su grado de voluntad será más alto. No obstante, hay circunstancias en las cuales, a pesar del costo que involucra la realización de la acción, el destinatario puede tener una fuerte voluntad para llevarla a cabo. Por ejemplo, si alguien quiere realizar una acción costosa con un fin altruista para beneficio de un tercero, o si la realización de la acción costosa depara beneficios para el futuro.

6. Grado de costo-beneficio: para el hablante, el destinatario y/o una tercera persona. En las órdenes prototípicas, el costo para el destinatario puede ser alto.

7. Grado de opcionalidad: el destinatario es libre de decidir sobre su curso de acción. Esta variable se vincula no solo con el tipo de acto de habla sino también con la distancia social y la formalidad del contexto. En una orden, cuanto más pequeña sea la distancia social y mayor la informalidad del contexto, mayor es el grado de opcionalidad. Las variables de costo-beneficio, opcionalidad, distancia social y poder ya habían sido consideradas por Leech (1983).

8. Grado de mitigación: grado en que la fuerza del acto de habla es suavizada.

9. Grado de poder: la posición relativa de hablante y destinatario en una jerarquía de autoridad. Cuanto más alto sea el grado de poder, más bajo será el grado de opcionalidad.

10. Grado de distancia social entre los interlocutores: la posición relativa de los participantes en un continuum de mayor a menor intimidad.

11. Grado de formalidad del contexto: el contexto puede ser formal y estructurado o informal y relajado. 
Aplicando la propuesta de Pérez Hernández (2001), en un análisis cualitativo de directivos en textos de opinión de medicina, fue posible identificar dos tipos de directivos no impositivos: propuestas y recomendaciones expertas (Ciapuscio y Gallardo 2017). Las primeras, que comprenden predicados de estado referidos a actividades cognitivas (actitudes, decisiones éticas) o tipos de comportamiento, se formulan en primera persona del plural, que incluyen al emisor como agente (debemos reflexionar/comprender) bregar por); el beneficiario es la sociedad en su conjunto. Estas propuestas expresan bajo grado de mitigación. Las recomendaciones expertas, dirigidas a un destinatario especializado (los médicos tratantes), se expresan con mayor grado de mitigación y desagentivación, en coincidencia con lo observado en artículos sobre salud de la prensa escrita (Gallardo 2004, 2005a y b, 2009). Además, ese análisis mostró que el estudio de los actos directivos requiere de la consideración del contexto textual.

Respecto de las estrategias de mitigación, se ha postulado que estas se emplean principalmente cuando el hablante vislumbra riesgos para la imagen del destinatario (Brown y Levinson [1978] 1987). No obstante, desde la sociopragmática, se han propuesto otras categorías de análisis con el fin de poner en evidencia que, en la comunicación, no siempre se pone en riesgo la imagen de los interlocutores. En esos casos, las estrategias corteses pueden operar reforzando la imagen social, que designa la autoestima que la persona asume para sí misma. Esta imagen puede ser de autonomía (autoafirmación) o de afiliación -el deseo de mantener lazos de cercanía y confianza con los demás miembros del grupo- (Bravo 1999, 2005). La imagen social también se vincula al rol, constituido por rasgos sociales más o menos permanentes, por ejemplo, en la comunicación médico-paciente, los hablantes activan sus respectivos roles de médico y paciente (Albelda Marco 2005; Hernández Flores y Rodríguez Tembrás 2018).

\section{CoRpus y MÉTODO}

Aquí se analizan seis interacciones orales entre médicos y pacientes que sufrieron infarto y fueron sometidos a una intervención cardiológica. Las grabaciones, que duran en promedio unos quince minutos, fueron tomadas en el Hospital El Cruce Néstor Kirchner, de la ciudad de Florencio Varela, en la provincia de Buenos Aires, Argentina. Este hospital es un centro de alta complejidad, que permite el acceso a una población de bajos recursos, y sin cobertura médica, de varias localidades del sudeste del conurbano bonaerense. Para acceder a la atención del hospital, los pacientes deben ser derivados de otros centros. 
Este estudio se centra en los actos de habla directivos; para ello, se consideran las once variables del MCI propuestas por Pérez Hernández (2001). Asimismo, el tipo de acciones o actividades que se proponen, para lo cual se realiza un análisis léxico-gramatical de los verbos o nombres deverbales que designan la acción o el comportamiento propuesto, considerando el tipo de verbo -de acción, estado o proceso- y el tipo de complemento, por ejemplo, nombres abstractos o concretos.

\section{ANÁlisis de los teXtos}

En las interacciones médico-paciente aquí analizadas, el médico formula principalmente actos de habla directivos de distinto tipo, acompañados de explicaciones, con el fin de dar al paciente las indicaciones necesarias para que pueda retirarse del hospital. De acuerdo con el contenido temático, los propósitos perseguidos y los tipos de actividades que se proponen, en las interacciones se pueden identificar cuatro etapas:

1. Apertura con entrega de la historia clínica

En esta etapa, el médico entrega la epicrisis o resumen de la historia clínica y describe el tipo de información que contiene, destacando su importancia para las futuras consultas que realice el paciente. Asimismo, recomienda ciertas acciones de rutina como guardar el informe y sacar copias para llevar a la consulta.

2. Indicación de la medicación

El médico indica en detalle al paciente cómo debe tomar cada medicamento, forma y horario. Asimismo, dado que el hospital provee esos medicamentos al paciente, este deberá realizar una serie de acciones de rutina, como guardar los envases y devolverlos, para que el hospital le provea la medicación adicional.

3. Indicación de los comportamientos a seguir en la casa

En esta etapa el médico da indicaciones sobre la alimentación, los hábitos, como el cigarrillo, y las actividades cotidianas como el trabajo, los esfuerzos y las caminatas.

4. Cierre

Una vez ofrecidas todas las indicaciones necesarias, la interacción finaliza, y el médico se despide del paciente.

En todas las etapas, los médicos formulan directivos, con distintos propósitos; y los pacientes responden confirmando su comprensión de las indicaciones y, en general, no realizan preguntas. 


\subsection{Tipos de directivos}

A partir del contenido proposicional (tipo de predicados y campo de aplicación de las acciones y actitudes propuestas), se identifican cuatro grupos de directivos:

1. Directivos que focalizan la atención.

2. Directivos que proponen acciones de rutina.

3. Directivos que proponen acciones y actitudes acerca de los medicamentos.

4. Directivos que proponen acciones y actitudes acerca de la dieta y los comportamientos diarios.

TABLA 1. Contenido proposicional de los directivos: campo de aplicación y tipos de predicados

\begin{tabular}{|c|c|c|}
\hline $\begin{array}{c}\text { Campo } \\
\text { de aplicación }\end{array}$ & $\begin{array}{c}\text { Tipos } \\
\text { de predicados }\end{array}$ & Verbos \\
\hline $\begin{array}{l}\text { Focalización } \\
\text { de la atención } \\
\text { en la interacción }\end{array}$ & $\begin{array}{l}\text { Procesos mentales } \\
\text { de percepción } \\
\text { y cognición }\end{array}$ & $\begin{array}{l}\text { Escuchar / mirar / fijarse } \\
\text { recordar / no olvidar / } \\
\text { tener en cuenta / } \\
\text { tener en mente / } \\
\text { entender / comprender }\end{array}$ \\
\hline Acciones de rutina & Acción & $\begin{array}{l}\text { Guardar / no tirar (envases) } \\
\text { traer / llevar / } \\
\text { Poner (algo en una bolsa) }\end{array}$ \\
\hline $\begin{array}{l}\text { Administración } \\
\text { de medicación }\end{array}$ & $\begin{array}{l}\text { Comportamiento } \\
\text { Estado }\end{array}$ & $\begin{array}{l}\text { Tomar / comenzar / } \\
\text { empezar a tomar } \\
\text { Seguir el tratamiento (adherir) } \\
\text { Ser regular }\end{array}$ \\
\hline $\begin{array}{l}\text { Dieta } \\
\text { y comportamientos } \\
\text { diarios }\end{array}$ & $\begin{array}{l}\text { Comportamiento } \\
\text { Movimiento } \\
\text { Elección } \\
\text { Acción }\end{array}$ & $\begin{array}{l}\text { Comer / fumar } \\
\text { caminar / ir a trabajar } \\
\text { prescindir / evitar (grasas, sal) } \\
\text { desgrasar / reemplazar / cocinar }\end{array}$ \\
\hline
\end{tabular}

Los directivos tienden a realizarse de forma diferente según el campo de aplicación de las acciones y actitudes propuestas (tipos de predicados). Se puede conjeturar que ello se debe a que, en cada grupo de predicados, se modifican para el destinatario las variables de costo-beneficio, voluntad para realizar la acción, y el grado de opción (ver Tabla 2). 
TABLA 2. Campo de aplicación de directivos y variables del Modelo Cognitivo Idealizado

\begin{tabular}{|c|c|c|c|c|}
\hline $\begin{array}{l}\text { Variables } \\
\text { MCI }\end{array}$ & $\begin{array}{l}\text { Focalización } \\
\text { atención }\end{array}$ & Rutina & Medicación & $\begin{array}{c}\text { Dieta } \\
\text { y comportamiento }\end{array}$ \\
\hline Tipo de agente & $\begin{array}{l}\text { El destinatario } \\
\text { (paciente) }\end{array}$ & $\begin{array}{l}\text { El destinatario } \\
\text { (paciente) }\end{array}$ & $\begin{array}{l}\text { El destinatario } \\
\text { (paciente) }\end{array}$ & $\begin{array}{l}\text { El destinatario } \\
\text { (paciente) }\end{array}$ \\
\hline Tiempo & Presente & Futuro & Futuro & $\begin{array}{l}\text { Futuro } \\
\text { (largo plazo) }\end{array}$ \\
\hline $\begin{array}{l}\text { Capacidad } \\
\text { del agente }\end{array}$ & Alta & Alta & Alta & No se sabe \\
\hline $\begin{array}{l}\text { Voluntad } \\
\text { del hablante }\end{array}$ & Alta & Alta & Alta & Alta \\
\hline $\begin{array}{l}\text { Voluntad } \\
\text { del destinatario }\end{array}$ & Alta & No se sabe & Alta & Media \\
\hline Costo & Bajo D & Bajo D & Bajo D & Alto D \\
\hline Beneficio & Alto H y D & Alto $\mathrm{H}$ & Alto D & Alto D \\
\hline $\begin{array}{l}\text { Grado } \\
\text { de opción }\end{array}$ & Bajo & Bajo & Bajo & Alto \\
\hline $\begin{array}{l}\text { Poder } \\
\text { del emisor }\end{array}$ & Alto & Alto & Alto & Alto \\
\hline Formalidad & Alta & Alta & Alta & Alta \\
\hline $\begin{array}{l}\text { Grado } \\
\text { de Mitigación }\end{array}$ & - & Baja & Baja & Alta \\
\hline Distancia social & Alta & Alta & Alta & Alta \\
\hline $\begin{array}{l}\text { Acciones / } \\
\text { actitudes } \\
\text { propuestas }\end{array}$ & $\begin{array}{l}\text { Percepción } \\
\text { y cognición }\end{array}$ & Acción & $\begin{array}{l}\text { Comportamiento } \\
\text { Estado }\end{array}$ & $\begin{array}{l}\text { Comportamiento } \\
\text { Movimiento } \\
\text { Elección / Acción }\end{array}$ \\
\hline
\end{tabular}

$\mathrm{Al}$ considerar la forma léxico-gramatical que adoptan las expresiones directivas, distinguimos siete categorías que se pueden ubicar en un continuum de mayor a menor grado de imposición. Estas categorías se establecen considerando las desinencias verbales (modo, tiempo, persona y número, formas verbales no personales); y el significado del verbo, verbos de modalidad deóntica -obligación, prohibición y permiso- y verbos de actitud intencional (Giammatteo 2014). 
1. Oraciones imperativas, en modo imperativo, en afirmativo o negativo (prohibición). Representan el mayor grado de imposición, y el menor de mitigación y de opción: por ejemplo, escúcheme, no lo tome, empiece, no vaya.

2. Modales deónticos: tener que y hay que más infinitivo, también la construcción lo + adjetivo con valor deóntico (lo importante es). Todas estas formas presentan un grado menor de imposición que el imperativo, pero restringen la opcionalidad del destinatario.

3. Oraciones declarativas, en modo indicativo en presente o futuro próximo (verbo $i r+$ infinitivo), en segunda persona del singular o primera del plural, que describen las acciones como habituales. Estas expresiones, en el contexto en cuestión, si bien poseen la fuerza ilocutiva de aserciones, se interpretan con una modalidad deóntica, como acciones a realizar. Este empleo del indicativo tendría un grado menor de imposición que el imperativo o los verbos de obligación. No obstante, considerando que son formuladas por el médico en el contexto del alta hospitalaria, pueden presentar bajo grado de opción.

4. Verbos en infinitivo independiente, por ejemplo: evitar los fiambres, caminar. Los infinitivos independientes pueden poseer un valor imperativo (Hernanz Carbó 1999), por ejemplo, en recetas de cocina o anuncios en espacios públicos, por ejemplo: No fumar, No estacionar, No pisar el césped.

5. Verbos de actitud intencional, en imperativo o infinitivo: tratar de más infinitivo, con el significado de procurar, que implica la necesidad de realizar un cierto esfuerzo para el logro de un fin. Pueden aparecer en infinitivo o en imperativo.

6. Empleo de la primera persona del plural, en modo indicativo, para referir a la acción que se propone, por ejemplo: la vamos a tomar, vamos a ir aprendiendo.

7. Verbos de modalidad deóntica que indican permiso: poder, permitir, admitir, dejar.

En líneas generales, los directivos que incluyen predicados de percepción y cognición y se formulan para focalizar la atención del destinatario, se realizan en imperativo, con el grado más bajo de mitigación. Los que proponen actividades de rutina y los que indican la medicación, presentan un grado mayor de mitigación; mientras que los que indican la dieta y comportamiento alcanzan el grado más alto de mitigación. Estos últimos se realizan mediante la frase verbal de actitud intencional tratar de más infinitivo, o el imperativo, en el caso de que la acción que se propone implique un bajo costo para el destinatario, por ejemplo: pida ayuda, no haga fuerza, no vaya a trabajar. Se muestran las frecuencias en las Tablas 3 y 4. 
TABLA 3. Frecuencia de los directivos según forma léxico-gramatical

\begin{tabular}{|c|c|c|c|c|c|c|c|c|}
\hline $\begin{array}{l}\text { Tipos de } \\
\text { predicados }\end{array}$ & Imperativo & Tener que & Indicativo & Infinitivo & Tratar de & $\begin{array}{c}1 .^{\text {a }} \text { pers. } \\
\text { plural }\end{array}$ & Permiso & Total \\
\hline $\begin{array}{l}\text { Percepción } \\
\text { y cognición }\end{array}$ & $\begin{array}{c}25 \\
83,6 \%\end{array}$ & $\begin{array}{c}2 \\
6,0 \%\end{array}$ & - & $\begin{array}{c}3 \\
10 \%\end{array}$ & - & - & - & $\begin{array}{r}30 \\
100 \%\end{array}$ \\
\hline Rutina & $\begin{array}{c}23 \\
32,3 \%\end{array}$ & $\begin{array}{c}10 \\
14,0 \%\end{array}$ & $\begin{array}{c}30 \\
42,5 \%\end{array}$ & $\begin{array}{c}8 \\
11,2 \%\end{array}$ & - & - & - & $\begin{array}{c}71 \\
100 \%\end{array}$ \\
\hline Medicación & $\begin{array}{c}8 \\
12,7 \%\end{array}$ & $\begin{array}{c}20 \\
31,7 \%\end{array}$ & $\begin{array}{c}32 \\
50,8 \%\end{array}$ & $\begin{array}{c}3 \\
4,7 \%\end{array}$ & - & - & - & $\begin{array}{r}63 \\
100 \%\end{array}$ \\
\hline Dieta & $\begin{array}{c}8 \\
7,7 \%\end{array}$ & $\begin{array}{c}9 \\
8,6 \%\end{array}$ & $\begin{array}{c}7 \\
6,7 \%\end{array}$ & $\begin{array}{c}34 \\
32,7 \%\end{array}$ & $\begin{array}{c}34 \\
32,7 \%\end{array}$ & $\begin{array}{c}4 \\
3,8 \%\end{array}$ & $\begin{array}{c}8 \\
7,7 \%\end{array}$ & $\begin{array}{c}104 \\
100 \%\end{array}$ \\
\hline Total & 64 & 41 & 69 & 48 & 34 & 4 & 8 & 268 \\
\hline
\end{tabular}

TABLA 4. Frecuencia de recomendaciones y prohibiciones acerca de dieta y comportamiento

\begin{tabular}{lccccccc}
\hline $\begin{array}{c}\text { Directivos } \\
\text { acerca } \\
\text { de la dieta }\end{array}$ & Imperativo & Tener que & Indicativo & Infinitivo & Tratar de & $\begin{array}{c}\text { 1. }{ }^{\mathrm{a}} \text { pers. } \\
\text { plural }\end{array}$ & Permiso \\
\hline \multirow{2}{*}{ Consejos } & 3 & 5 & 7 & 24 & 14 & 2 & 8 \\
& $37,5 \%$ & $55,5 \%$ & $6,7 \%$ & $70,6 \%$ & $41,2 \%$ & $50 \%$ & $7,7 \%$ \\
\hline \multirow{2}{*}{ Prohibiciones } & 5 & 4 & & 10 & 20 & 2 & - \\
& $62,5 \%$ & $44,4 \%$ & - & $29,4 \%$ & $58,8 \%$ & $50 \%$ & \\
\hline \multirow{2}{*}{ Total } & $\mathbf{8}$ & $\mathbf{9}$ & $\mathbf{7}$ & $\mathbf{3 4}$ & $\mathbf{3 4}$ & $\mathbf{4}$ & $\mathbf{8}$ \\
& $\mathbf{1 0 0 \%}$ & $\mathbf{1 0 0 \%}$ & $\mathbf{1 0 0 \%}$ & $\mathbf{1 0 0 \%}$ & $\mathbf{1 0 0 \%}$ & $\mathbf{1 0 0 \%}$ & $\mathbf{1 0 0 \%}$ \\
\hline
\end{tabular}

A continuación detallamos las características de cada uno de los tipos de directivos según el campo de aplicación de las actividades propuestas.

\subsubsection{Directivos para focalizar la atención}

Los directivos orientados a focalizar la atención del interlocutor aparecen a lo largo de la interacción, pero especialmente en la etapa de indicaciones sobre la medicación. Su contenido proposicional incluye verbos de percepción como mirar y escuchar, o verbos de cognición: acordarse, no 
olvidarse. En algunos casos se acompañan de deícticos, como el adverbio demostrativo acá, mediante el cual el hablante muestra un objeto (el envase de un medicamento) al destinatario, para asegurar la comprensión. Pueden considerarse como «metadirectivos», en términos de Mulder (1993), pues, aunque tienen un valor directivo, actúan de sostén de otro acto de habla. Se formulan en general en imperativo, sin mitigación y, por ende, sin opción para el agente. La intención del hablante es lograr una respuesta inmediata por parte del destinatario. Se ejemplifica a continuación:

(1) M: [hm'. $a c A^{1}$.. en el apartado de tratamiENto están las indicaciones. $\mathrm{P}$ : perfecto $\mathrm{mhm}$

M: [si'. de la medicación que tiene que tomar=y cómo la tiene que tomar. PRIMERO. el clopidogrel. que es esta caJIta de aCÁ. fijeSE que los medicamentos tienen dos nOmbres. el comerciAL. que puede ser.. HAy montÓNes. depende del laboratOrio que l=haga. y eso [P3]

(2) M: bueno mIre... aCÁ. está anotado qué es lo que tiene que tomar,.. [sí'... nosOtros le vamoh a dar la medicación. tiEne que tomar.. SAbe leer. [no' Una aspirina. por día.. a las ocho de

P: $\quad$ sisi

M: la mañana. [ve que acá le puse UN comprimido a las ocho entre paréntesis' eso es que lo

P: si

M: tiene que tomar a las ocho de la mañana. NO cada ocho horas [eh'. [P5]

En los ejemplos (1) y (2) el médico indica la medicación al paciente focalizando su atención ya sea en el envase del medicamento o en el texto (epicrisis) donde se consignan esas indicaciones. La información llega al paciente por partida doble, está anotada en la epicrisis, y el médico la refuerza en forma oral, haciendo referencia al texto escrito, que es la única indicación que tendrá el paciente cuando esté ya en su casa. La insistencia en focalizar la atención junto con los refuerzos, como reformulaciones y repeticiones para asegurar la comprensión, pueden responder al propósito del médico, en su rol, de garantizar que se cumpla la acción, teniendo en cuenta la importancia de cumplir con la medicación para un paciente que fue sometido a una cirugía cardiovascular. Por ejemplo, en (2), al indicar el horario para tomar la medicación, el médico aclara que es a las ocho de la mañana, y no cada ocho horas, presuponiendo una interpretación errónea por parte del paciente. Además, el médico quiere asegurarse de que el paciente sabe leer, teniendo en cuenta que los pacientes que acceden al hospital en cuestión poseen bajo nivel de escolarización.

${ }^{1}$ Se destacan en cursiva los directivos, los deícticos y cualquier otro componente que se considere relevante. 
Los metadirectivos también se formulan en la etapa de cierre, en la despedida. En algunas de las interacciones, el médico le dice al paciente: ;cuidáte!; esta es una fórmula coloquial que sugiere proximidad y familiaridad entre los interlocutores, y también afecto. El verbo pronominal cuidarse se define como «Mirar por la propia salud, darse buena vida». Dado que el beneficio es para destinatario, no se trata de una orden sino de un consejo. El hecho de que se formule sin mitigación indica intimidad o distancia social baja (Pérez Hernández 2001). En este caso, el médico posiblemente emplee la forma amistosa como un intento por disminuir la asimetría, y reforzar la imagen de afiliación, como se muestra en el ejemplo (3).

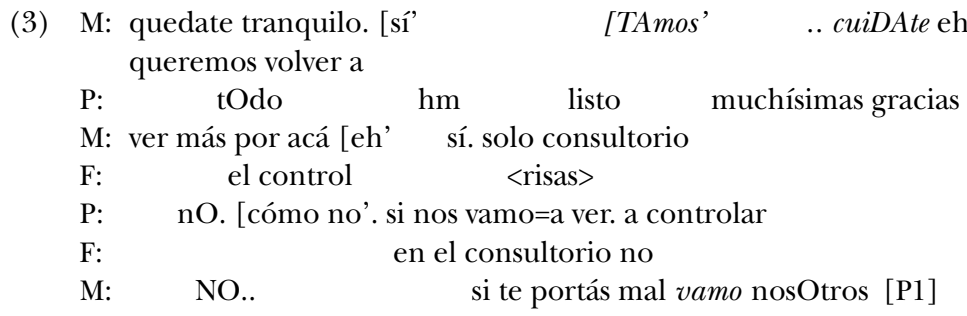

Como se puede ver en el ejemplo (3), se formulan dos directivos en imperativo: un consejo reconfortante -quedáte tranquilo- (Gallardo, 2005), y una orden aparente (cuidate). Ambos directivos pueden interpretarse como acciones de cortesía positiva, destinados a reforzar la proximidad y la solidaridad entre los interlocutores. La misma función cumple la expresión de deseo: no te queremos volver a ver más por acá, que, en este contexto, se interpreta como una broma, de hecho hay risas. Es un acto de habla indirecto en el que se realizan simultáneamente dos actos de habla, uno primario, el no literal (el deseo de que el paciente se recupere), y uno secundario, el literal, que en este caso es una hipérbole, el deseo de no ver más al paciente (Mulder 1993). Al final el médico formula también una especie de amenaza: si te portas mal, vamos nosotros, que se entiende como broma. De este modo, el emisor refuerza la imagen de afiliación del paciente, haciendo que este se sienta satisfecho y bien atendido. Al mismo tiempo, remarca la obligación del paciente de acatar las indicaciones: portarse mal significa no acatarlas. Por su parte, el paciente se muestra agradecido y confirma que volverá a ver al médico en el consultorio.

\subsubsection{Directivos que proponen acciones de rutina}

Denominamos acciones de rutina a aquellas como guardar un documento, o devolver al hospital los envases vacíos de medicamentos; son actividades 
con bajo costo para el agente, pues no demandan tiempo ni esfuerzo. El contenido proposicional de estos enunciados incluye ciertos indicadores, como el dativo de interés y la expresión por favor, como lo muestran los ejemplos (4) y (5):

(4) M: [NO MEtire los BLIster vAcíos. CUANdo me viene a ver A MI. que va a ser el juEves veintiSIEte

M: Osea el juÉves de la semana que vIEne No. el Otro. el jueve/o sea la semana que

M: nO. el Otro jueves me tIEne que venir a ver. me trae tOdo. lo vacío y lo que le sObró. [P3]

(5) M: ahora le voy a explicar porque ya hablamos con él. y ESte es la epiqui/ esto es la epicrisis que es un resumen comPLEto de la historia clínica que forma. quÉ fue lo que le paSÓ. quéfue lo que le hicimo y cómo se va a casa. [esTAmos' tiene que guardarselo/ por favor

F: $\quad$ si

M: porque es un docuMENto. si pasa algo por ejemplo en alguna ocasión duele el

F: sí no sísísí

M: pecho usted. se aCERca CLAro tiene que traerlo así el el colEga que lo atiende

P: $\quad$ claro

F: $\quad$ tiene que traerlo

M: ya se ubica de que estamos tratando a usted SÍ. entonces esto lleveselo por favor [P1]

En el ejemplo (4), el pronombre dativo de interés y, en (5), la expresión por favor, indican al hablante como beneficiario de la acción, presentando el directivo como un pedido y se interpretan como una muestra de la alta voluntad del médico para que se realice la acción. Esta voluntad se evidencia también mediante las repeticiones y reformulaciones para asegurar la comprensión. La ausencia de mitigación puede deberse a que se supone bajo costo para el agente, y también un beneficio: de hecho, si el paciente devuelve los envases vacíos de los medicamentos, podrá obtener más medicamentos para continuar el tratamiento. Lo mismo se puede decir respecto de la explicación acerca de la importancia de la epicrisis como documento y la necesidad de que el paciente la lleve consigo en todas sus consultas. Cabe señalar que en esta interacción se halla presente, además del paciente, un familiar de este, quien repite el directivo formulado por el médico (tiene que traerlo), reforzando las palabras del especialista y mostrando que se ha comprendido el acto de habla. Son acciones de refuerzo que contribuyen a mostrar que se está cooperando con el discurso. 


\subsubsection{Directivos para indicar la medicación}

Dado que los pacientes deben tomar varios medicamentos, el médico indica los fármacos, la dosis, los horarios y el orden en que deben tomarse. En este sentido, se observa el empleo de los verbos aspectuales incoativos comenzar e iniciar. Estas indicaciones, que son para el beneficio del agente, se formulan con bajo grado de mitigación y de opción, lo cual se vincula con la importancia que ello tiene para la salud del paciente y el riesgo que implica no cumplirlo. En muchos casos, la acción que debe realizar el paciente se expresa en modo indicativo, en segunda persona del singular y en tiempo presente o futuro próximo, mediante la frase verbal con el verbo ir más infinitivo.

(6) M: empezás con Estos. TOdos los días. y te tomás esto que se llama clopidoGREl todos lo/todas las mañanas junto con esto,. con el doctor RosEnde vas a hablAr con él. a ver cuánto tiempo lo vas a tomar. [sí’ eso lo vas a dialogar con él. pero el tema=es el siguiEnte. es

P: $\quad \mathrm{mhm}$

M: que tenés que tomar las dos medicaciones. a la mañana juNtas. no hay problEma. antes del

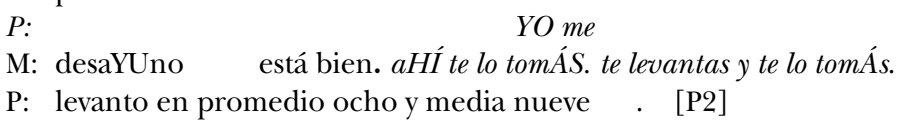

(7) M: al MEnos. lo va a tener que tomar un año. un año. los Otros SI son de por vida. ESte. un año. lo importAnte es que DENtro de ese año tiene que tomarlo religiOsamente todos los días.

F: $\quad$ si

$$
\text { si }
$$

M: uno por día, acÁ tiene trEinta comprimidos, y acá yo le hice una receta para que lo compre por SESEnta comprimidos. [por qué'. porque le sale más barato comprando

F: en (?un) horario de que,

F2: eso es uno por día

M: de sesENta que de trEInta, y le dura más tiempo. [si' eh. así que para que no se quede sin medicación. si bien yo la voy a ver antes. vaya consiguiéndolo y tÉngalo siempre ahí. [si’ ESte por un lado AHOra el RESto de los medicamentos que vA A tomAr. las SIMvastatinas

P: bueno $\mathrm{mhm}$ si..

M: para bajar el colesterol, viene de vEInte miligrAmos. usted va a tomar DOS. [P3]

En los ejemplos (6) y (7) se observa variación en la realización de los directivos: se emplea el indicativo en presente y futuro próximo, el imperativo y también las formas verbales con el modal deóntico tener que. El grado de opción es bajo, incluso en las formas de indicativo. El médico manifiesta alta voluntad en que el paciente siga sus indicaciones, y ello se evidencia en 
las repeticiones, que operan como refuerzo, por ejemplo: lo va a tener que tomar un año, un año, o el adverbio religiosamente, con función enfática.

Los actos de habla en modo indicativo presente o futuro próximo son asertivos; no obstante, en el contexto de la interacción entre médico y paciente, se interpretan como directivos. El empleo del indicativo presente en lugar del imperativo no parece constituir una mitigación, porque no brinda al destinatario mayor grado de opción; antes bien, podría responder al propósito de disminuir el compromiso por parte del hablante, o fuente modal.

Estos directivos sobre la medicación se acercan más a las órdenes que a los consejos, pues los consejos prototípicos se realizan con alto grado de mitigación y alto grado de opción, en particular si la distancia social es elevada (Pérez Hernández 2001). De todos modos, cuando el médico prescribe la medicación y, en particular, en el momento del alta hospitalaria de un paciente que ha sufrido infarto, el consejo puede ser demandado e, incluso, esperado por el paciente, por lo cual, su imagen no estaría en riesgo; además, el consejo sin mitigación en este caso puede reforzar la imagen de afiliación del paciente, al sentirse bien atendido (Hernández Flores 2013; Hernández Flores y Rodríguez Tembrás 2018).

\subsubsection{Directivos para indicar la dieta y comportamientos diarios}

En la etapa final de la interacción, el médico formula expresiones de obligación, de prohibición y de permiso acerca de la dieta y las actividades que el paciente podrá realizar en su hogar, luego de salir del hospital. Los directivos de prohibición se realizan mediante la negación sintáctica (adverbio de negación) o la negación léxica, a través de predicados como evitar y prescindir, o la preposición sin, que indica falta o privación.

En esta etapa, los directivos se realizan con mayor grado de mitigación en comparación con los vinculados a otras áreas de aplicación. Por ejemplo, es alta la frecuencia de la construcción formada por los verbos de actitud intencional tratar de, en imperativo; también la construcción con el verbo deóntico tener que en imperativo más el verbo intentar, con significado similar a tratar de y procurar. Este tipo de construcción, considerada por Mulder (1998) como un metadirectivo, cumple, en nuestra opinión, una función mitigadora, pues aumenta el grado de opción del destinatario, y da a entender que, si bien las acciones que se proponen son beneficiosas para el destinatario, representan un alto costo, por constituir un cambio en los hábitos. De este modo, la mitigación refuerza la imagen de autonomía del paciente. Por otra parte, los directivos se encuentran apoyados por aserciones explicativas, mediante las cuales el médico justifica el consejo. 
(8) M: sî' otra cosa tratá de comer sin sal' mhm. para

P: LIsto listo CLAro

M: no=aumentar la preSIÓN. y que=el corazón se tenga que exigir un poco más' [sí'. sobre todo al no coMER. e: cosas: como. paPItas chiSItos maní los CALdos. caldos a/los caldos artifiCIAles (?...)

P: sí no:. eso no: sí no:P: todo de verdura tiene que ser MUcho sodio

M: esos tienen. MUcho contenido de sodio los JUgos artificiales M:

también. e: así que traTAR de evitar esos alimentos que tienen mucha sal Y coman sin grasa.. [P4]

En (8), la prohibición de ingerir sal, además de representar un esfuerzo para el destinatario, podría afectar su imagen de autonomía (Bravo 1999), lo cual explica el empleo de la mitigación mediante el metadirectivo tratar $d e$. El médico enumera los alimentos que el paciente tendrá que evitar, dando la denominación coloquial de cada uno (papitas, chizitos), reforzando la imagen de afiliación. También apoya el consejo mediante razones que justifican esa prohibición, repitiendo y enfatizando el cuantificador mucho, para dar cuenta del exceso de sodio que poseen estos alimentos ultraprocesados. De este modo, el especialista evidencia su alto grado de voluntad para lograr que el destinatario lleve adelante las acciones propuestas. El paciente confirma que ha entendido los consejos del médico, señalando que no va a ingerir caldos artificiales, sino alimentos sanos (todo de verdura). En este caso, el cuantificador todo intensifica su grado de voluntad para cumplir con los consejos.

En esta etapa, el médico emplea diferentes procedimientos de mitigación. En algunos casos, en lugar de prohibir la ingesta de determinado alimento, formula una advertencia, por ejemplo: Tener mucho cuidado con los fiambres; cuidarse de (determinados productos). Otro procedimiento de mitigación es el empleo de la primera persona del plural, una forma pseudoinclusiva, que simula incluir al emisor como agente; consiste en una enálage de persona en que se emplea la primera del plural en lugar de la segunda del singular (Caffi 1999). También se formulan permisos condicionados: $V a$ a poder comer de todo siempre que sea sin sal; en este caso la prohibición pasa a un segundo plano, y se convierte en una condición para disponer de la libertad de «comer de todo». Un permiso es mucho menos impositivo que una prohibición, de modo que formular una prohibición como una condición para disponer de un permiso es una forma de mitigación y de refuerzo de la imagen de autonomía del destinatario.

Las indicaciones para la dieta también se formulan en presente de indicativo, segunda persona singular, en lugar de imperativo, como lo vimos en otras etapas, en que se enumeran las acciones a llevar a cabo, como en una receta de cocina: Le saca la grasa al pollo, le saca la piel y lo cocina bien. 
(9) M: [sí... [sí'. por eso es importante que. una de las grandes cosas que hemos charlado varias

P: claro

M: veces con vos ya el cigarrIllo. tabas fumando trEINta cuarenta cigarrillos por día. miRÁ. a veces no es tan fácil. lo que YO te digO es que:: no nos=ocultes nada más eso digamos. si vos me decís mirá la verdad que no puEdo chárlalo con nosotro. y vemos qué podemo=hacer .. <respira hondo $>$ a veces no es tan sencillo pero por favor. es muy importante que DEjes de fumAr. [sí'. e::. y que si nO podés que charles con nosotro y: y vemos como: podemos hacer un tratamiENto algo. peRO pero es $\mathrm{mUy}$ importante esto para vos [sí' es mUY importante

P: no se puede dejar

M: pORque:=es un factor de rIESgo mUy pesado,.. fume la

F:

M: verdad es esa. [sí'. la verdad es esa. si no podÉs mirá vení deCIno. e $e$ y nosotro:.. [P1]

En el ejemplo (9), al emplear la expresión por favor, el médico refuerza el consejo y lo formula como un pedido, evidenciando su alto grado de voluntad para que se cumpla la acción. Además, apoya sus directivos con comentarios de empatía, por ejemplo al decir que no es tan fácil dejar de fumar, y más adelante reformula la frase a veces no es tan sencillo. Es una forma de manifestar reconocimiento al esfuerzo que representa para el paciente privarse del cigarrillo. El gesto del profesional de respirar hondo (indicado en la transcripción) sugiere su preocupación por el tema. Al mismo tiempo, este refuerza el directivo mediante repeticiones: Es muy importante para vos, sí, es muy importante. Y a continuación formula una explicación para justificar el consejo: es un factor de riesgo muy pesado; al mismo tiempo, adopta un tono confidencial, al pedirle al paciente que converse con él si no puede dejar de fumar. Por su parte, un familiar que está presente también formula un directivo (que no fume escondido), mostrando su acuerdo con el especialista y también su voluntad de acompañar al paciente para poder cumplir con el consejo.

En el corpus, los médicos no emplean tecnicismos, sino que usan un registro coloquial. Recordemos, de paso, que el hospital en cuestión recibe a pacientes de muy bajos recursos y bajo nivel de escolarización. Asimismo, cabe destacar que, en el fragmento (9), el médico emplea algunos rasgos del habla coloquial rioplatense, como la elisión de una sílaba (tabas, por estabas), o la elisión de la 's' final del dativo de primera persona (decíno, por decínos; nosotro por nosotros). Estos usos pueden interpretarse como un intento de acortamiento de la distancia social con el paciente en un tema delicado como el dejar de fumar; y también como acciones de cortesía positiva, que refuerzan la imagen de afiliación, y los lazos entre los interlocutores. De este modo el médico se acerca al paciente en un rol más cercano al de un amigo, al que se le pueden contar sus dificultades. Se trata de lo 
que Cepeda (2005) denomina «voz de la cortesía de solidaridad» o «voz empática», mediante la cual el profesional muestra interés y reconocimiento del paciente como persona.

Hasta aquí nos hemos concentrado en los actos de habla que formulan los médicos y poco hemos dicho acerca de los pacientes. En todos los casos, la participación de estos consiste en expresar frases de asentimiento, evidenciando que se han comprendido las palabras del especialista. La conducta de los familiares, en los casos en que se encuentran presentes, es similar: asentimiento y alineación con las palabras del profesional. En cierta manera, esas expresiones constituyen una manifestación de apoyo o aceptación de los consejos recibidos, y de aceptación y respeto del rol del médico. También es una forma de ser cortés (Albelda Marco 2005).

\section{Comentarios Finales}

Aquí hemos analizado los actos directivos que se formulan durante el alta hospitalaria y hemos identificado cuatro tipos según las actividades que se proponen. Los directivos difieren en el grado de mitigación y opción ofrecida al destinatario, según el costo-beneficio de las acciones propuestas. En líneas generales, los directivos que incluyen predicados de percepción y cognición, y focalizan la atención del destinatario se realizan en imperativo, con bajo grado de mitigación. Podrían interpretarse como pedidos, por la presencia de expresiones que se asocian con pedidos prototípicos; en tal sentido, es bajo el grado de opción del destinatario para rehusar un pedido del médico. Los directivos de actividades de rutina e indicación de medicación se realizan con un grado mayor de mitigación que los focalizadores de atención. Al indicar la medicación, los profesionales no mitigan sus directivos, lo cual ofrece al destinatario un grado de opción bajo o nulo: el paciente, luego de un infarto y una cirugía cardiovascular, no puede obviar la toma de medicamentos, pues de ello depende su vida.

Los directivos con más alto grado de mitigación son los que indican la dieta y el comportamiento, que pueden implicar un alto costo para el agente. Estos se realizan principalmente mediante la construcción de actitud intencional tratar de más infinitivo, con el significado de 'intentar' o 'hacer un esfuerzo'. El imperativo, que aparece en pocos casos, se formula en propuestas que implican bajo costo para el agente, como pedir ayuda o no ir a trabajar. Se destaca el uso del modo indicativo en presente o futuro próximo en reemplazo del imperativo para indicar acciones a realizar. El sentido literal de los enunciados es enumerar acciones presentadas como habituales o futuras, pero el propósito es indicar al destinatario lo que debe realizar. Este empleo del indicativo es una forma de mitigación, pero no brinda al destinatario mayor grado de opción; tal vez su empleo responda 
al objetivo de disminuir el compromiso del hablante, en comparación con el imperativo o el verbo deóntico tener que.

Como ya señalamos, el alta de la internación es un momento de gran importancia en el ámbito hospitalario, en especial luego de intervenciones quirúrgicas, debido a que los profesionales deben asegurarse de que los pacientes puedan seguir las indicaciones en su hogar e incorporarse a una vida normal, y así evitar el reingreso a la institución. Respecto de las interacciones de nuestro corpus, incluyen a pacientes que fueron sometidos a una cirugía cardiovascular de alta complejidad, que implica serios cuidados luego del alta, lo que explica el esfuerzo de los médicos por asegurarse de que los pacientes comprendan las indicaciones y las acaten. Hemos observado que, durante la interacción, los médicos han modulado la fuerza de sus directivos con el fin de atender tanto a la imagen positiva como a la negativa del paciente; y desplegaron recursos de cortesía positiva, mostrando proximidad y solidaridad, y reforzando la imagen de afiliación (Hernández Flores y Rodríguez Tembrás 2018). Asimismo, emplearon recursos de mitigación en los directivos vinculados a aspectos más personales como los hábitos y la alimentación, reforzando así la imagen de autonomía de los pacientes. Estos, por su parte, dieron respuestas de asentimiento y confirmación de las palabras del especialista, lo cual permite inferir que la imagen de aquellos no estaba siendo amenazada. Además, estos directivos se encuentran apoyados por información que fundamenta las indicaciones. En síntesis, se puede afirmar que la forma en que el médico formula los directivos en el momento del alta hospitalaria parece vincularse al costo-beneficio de las actividades propuestas y a su voluntad de que el paciente las lleve a cabo. Además, los profesionales adaptan sus enunciados a la situación del paciente, no empleando tecnicismos sino un registro coloquial, que por un lado facilita la comprensión del contenido y, por otro, fortalece lazos de solidaridad. También emplean estrategias de intensificación, mediante repeticiones, reformulaciones y pedidos de confirmación de que se ha entendido el mensaje.

Si bien este es un aporte a la caracterización de la interacción médicopaciente durante el alta hospitalaria (un género discursivo poco estudiado), hemos considerado solo casos de pacientes cardiovasculares que fueron sometidos a cirugía. En tal sentido, sería necesario realizar un análisis más amplio, con un corpus más extenso de interacciones orales, considerando, no solo pacientes con distinto tipo de condición de salud, sino también pertenecientes a otros sectores sociales, con el fin de determinar si las características halladas pueden generalizarse para otros casos y otras situaciones, o tal vez sean propias de los médicos que tratan pacientes que han estado en una condición de salud extremadamente delicada ${ }^{2}$.

${ }^{2}$ Deseo agradecer a los árbitros anónimos por sus aportes, que permitieron mejorar en gran medida este trabajo. 


\section{BIBLIOGRAFÍA}

Ainsworth-Vaughn, Nancy (2003): «The Discourse of Medical Encounters». En Deborah Tannen, Heidi E. Hamilton, Deborah Schiffrin (eds.), The Handbook of Discourse Analysis, Cornwall: Blackwell Publishing, 453-469.

Albelda MARCO, Marta (2005): «El refuerzo de la imagen social en conversaciones coloquiales en español peninsular. La intensificación como categoría pragmática». En Diana Bravo (ed.), Estudios de la (des)cortesía en español. Categorías conceptuales y aplicaciones a corpora orales y escritos, Buenos Aires: Dunken, 93-118.

AlPER, Eric, Terrence O'MALLEY y Jeffrey GreENWALd (2017): «Hospital discharge and readmission». <https://www.uptodate.com/contents/hospital-dischargeand-readmission $>$.

Aronsson, Karin y Ullabeth SÄtterlund-Larsson (1987): «Politeness Strategies and Doctor-Patient Communication. On the Social Choreography of Collaborative Thinking», Journal of Language and Social Psychology 6/1, 1-27.

BAgHeri, Hossein, Noor Aireen IbraHIm y Hadina HabIL (2015): «The Structure of Clinical Consultation: A Case of Non-Native Speakers of English as Participants», Global Journal of Health Science 7/1, 249-260.

BAÑón HERnÁNDEZ, Antonio (2017): «Los modelos de interacción entre médico y paciente: Descripción y aplicación al contexto de las enfermedades poco frecuentes», Oralia 20, 13-43.

BRAVO, Diana (1999): «¿Imagen positiva vs. imagen negativa? Pragmática sociocultural y componentes de face», Oralia 2 , 155-184.

- (2005): «Categorías, tipologías y aplicaciones: hacia una redefinición de la cortesía comunicativa». En Diana Bravo (ed.), Estudios de la (des)cortesía en español. Categorías conceptuales y aplicaciones a corpora orales y escritos, Buenos Aires: Dunken, 21-52.

Brown Penelope y Stephen Levinson ([1978] 1987): Politeness: Some Universals in Language Usage, Cambridge: Cambridge University Press.

CAFFI, Claudia (1999): «On Mitigation», Journal of Pragmatics 31/7, 881-909.

CEPEDA, Gladys (2005): «Cortesía, imagen social y aceptación del mensaje terapéutico». En Diana Bravo (ed.), Estudios de la (des)cortesía en español. Categorías conceptuales y aplicaciones a corpora orales y escritos, Buenos Aires: Dunken, 163-187.

ČERnÝ, Miroslav (2007): «On the Function of Speech Acts in Doctor-Patient Communication». <http://www.phil.muni.cz/linguistica/art/cerny/cer-001.pdf>.

- (2010a): «Questioning and Responding. Practices in Medical Interviews Revisited», Ostrava Journal of English Philology 2, 67-86.

- (2010b): «Interruptions and Overlaps in Doctor-Patient Communication Revisited», Linguistica Online 11 (Miscellanea III), 1-20.

- (2014): «Empathy and Trust as Key Notions in English Medical Discourse», Journal of ELT and Applied Linguistics 2, 18-27. 
Ciapuscio, Guiomar, Andreína Adelstein y Susana Gallardo (2010): «El texto especializado: propuesta teórica y prácticas de capacitación académica y profesional en la Argentina». En Giovanni Parodi (ed.), Alfabetización académica y profesional en el siglo XXI: Leer y escribir desde las disciplinas, Barcelona: Editorial Planeta, 317-345.

- y Susana Gallardo (2018): «Actos de habla directivos en géneros de opinión de revistas médicas». En Roberto Bein et al. (eds.), Homenaje a Elvira Arnoux. Estudios de análisis de discurso, glotopolítica y pedagogía de la lectura y la escritura, Buenos Aires: Oficina de Publicaciones Filosofía y Letras-UBA, 97-114.

Cicourel, Aaron (1985): «Doctor-Patient Discourse», Discourse Analysis in Society, vol. 4, London: Academic Press, 193-202.

- (2005): «Bureaucratic Rituals in Health Care Delivery», Journal of Applied Linguistics 2/3, 357-370.

COUlTHARD, Malcolm y Margaret AshBy (1975): «Talking with the Doctor», Journal of Communication 1, 140-147.

Drass, Kriss A. (1982): "Negotiation and the structure of discourse in medical consultation», Sociology of Health and Illness 4/3, 320-341.

FISCHER, Sue (1984): «Doctor-patient communication: a social and micro-political performance», Sociology of Health and Illness 6/1, 1-29.

- y Stephen B. Groce (1985): «Doctor-patient Negotiation of Cultural Assumptions», Sociology of Health and Illness 7/3, 342-374.

Fleischman, Suzanne (2003): «Language and Medicine». En Deborah Tannen, Heidi E. Hamilton, Deborah Schiffrin (eds.), The Handbook of Discourse Analysis, Cornwall: Blackwell Publishing, 470-502.

GALlARDo, Susana (2004): «Realizaciones léxico-gramaticales de la recomendación en artículos de medicina en la prensa escrita», $R L A$ 42/1, 111-135.

- (2005a): «Pragmatic Support of Medical Recommendations in Popularization Texts», Journal of Pragmatics 37/6, 813-835.

- (2005b): Los médicos recomiendan. Un estudio de las notas periodísticas sobre salud, Buenos Aires: Eudeba.

- (2009): «La estructura ilocutiva y la distinción entre géneros discursivos». En Guiomar Elena Ciapuscio (ed.), De la palabra al texto. Estudios lingüisticos del español, Buenos Aires: Eudeba, 131-164.

Giammatteo, Mabel (2014): «La modalidad volitiva y los verbos de actitud intencional», Traslaciones 1/1, 116141.

Gras, Pedro y María Ángeles García (2010): «La delimitación de la recomenda-

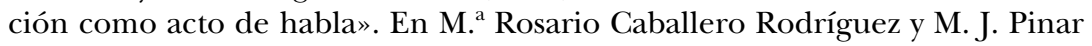
Sanz (eds.), Modos y formas de la comunicación humana, Cuenca: Publicaciones de la Universidad de Castilla-La Mancha, 681-688.

GüLICH, Elizabeth (2003): «Conversational Techniques used in Transferring Knowledge between Medical Experts and Non-experts», Discourse Studies 5/2, 235-263.

Haverkate, Henk (1984): Speech acts, speakers and hearers. Pragmatics and beyond 4, Ámsterdam: John Benjamins.

Heritage, John y Sue Sefi (1992): «Dilemmas of Advice: Aspects of the Delivery and Reception of Advice in Interactions between Health Visitors and First- 
Time Mothers». En Paul Drew y John Heritage (eds.), Talk at Work: Interaction in Institutional Settings, Cambridge: Cambridge University Press, 359-417.

Hernández Flores, Nieves (2013): «Actividad de imagen: caracterización y tipología en la interacción comunicativa», Soprag 1/2, 175-198.

- y Vanesa Rodríguez Tembrás (2018): «Estrategias de atenuación en el consejo médico», Spanish in Context 15/2, 325-345.

Hernanz Carbó, M. ${ }^{a}$ Lluïsa (1999): «El infinitivo». En Ignacio Bosque y Violeta Demonte (dirs.), Gramática descriptiva de la lengua española, Madrid: Espasa Calpe-Real Academia Española.

LAKOFF, George (1987): Women, fire and other dangerous things, Chicago: The University of Chicago Press.

LEECH, Geoffrey (1983): Principles of Pragmatics, London: Longman.

Montolío, Estrella y Anna LóPEz SAMANIEgo (2010): «Especificidades discursivas de los textos profesionales frente a los textos académicos: el caso de la recomendación profesional». En Giovanni Parodi (ed.), Alfabetización académica y profesional en el siglo XXI: Leer y escribir desde las disciplinas, Madrid: Editorial Planeta, 215-245.

Mulder, Gijs (1993): «¿Por qué no coges el teléfono? Acerca de los actos de habla indirectos», Diálogos Hispánicos 12, 181-208.

- (1998): «Un estudio empírico de los actos de habla directivos en español», Diálogos hispánicos 22, 237-276.

PÉREZ HERnÁNDEZ, Lorena (2001): Illocution and Cognition: A Constructional Approach, Logroño: Universidad de La Rioja.

Rodríguez Tembrás, Vanesa (2016): «Alternancia de lenguas como estrategia de actividad de imagen en la comunicación médico-paciente en un consultorio gallego", Textos en Proceso 2/1, 94-121.

Rush Kathy, Maryy KJORVEn y Rachelle Hole (2016): «Older Adults' Risk Practices From Hospital to Home: A Discourse Analysis», Gerontologist 56/3, 494-503.

SEARLE, John (1969): Actos de habla, Madrid: Cátedra.

Ten Have, Paul (1989): «The consultation as genre». En Brian Torode (ed.), Text and Talk as Social Practice, Dordrecht: Foris, 115-135.

Verstraete, Jean Cristophe (2005): «Scalar Quantity Implicatures and the Interpretation of Modality. Problems in the Deontic Domain», Journal of pragmatics, 37/9, 1401-1418.

West, Candace (1990): «Not Just 'Doctors' Orders': Directive-Response Sequences in Patients' Visits to Women and Men Physicians», Discourse and Society 1/1, 85-112. 


\section{APÉNDICE}

\begin{tabular}{ll}
\hline & \multicolumn{1}{c}{ CONVENCIONES DE TRANSCRIPCIÓN } \\
\hline, & Curva de entonación ascendente \\
\hline MAYÚSCULAS & Curva de entonación descendente \\
\hline$:$ & Elargasis \\
\hline$/$ & Interrupción perceptible, corrección \\
$=$ & Unión perceptible \\
\hline ... ... & Pausa: muy corta, corta, más extensa \\
\hline ?palabra) & Transcripción insegura \\
\hline
\end{tabular}

